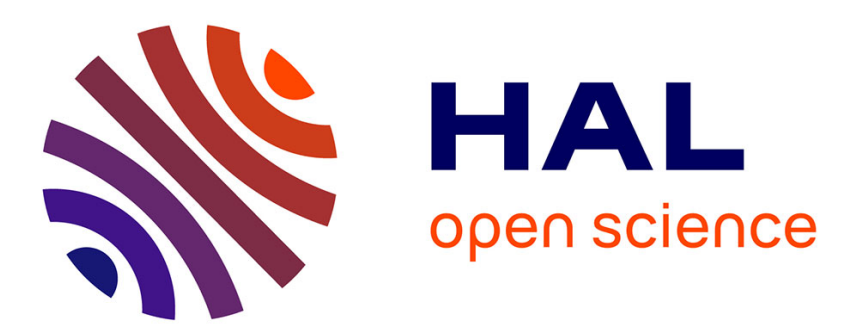

\title{
A switching technique for output feedback stabilization at an unobservable target
}

Lucas Brivadis, Ludovic Sacchelli

\section{To cite this version:}

Lucas Brivadis, Ludovic Sacchelli. A switching technique for output feedback stabilization at an unobservable target. CDC 2021 - 60th IEEE Conference on Decision and Control, Dec 2021, Austin, Texas, United States. pp.3942-3947, 10.1109/CDC45484.2021.9683664 . hal-03180479

\section{HAL Id: hal-03180479 \\ https://hal.science/hal-03180479}

Submitted on 25 Mar 2021

HAL is a multi-disciplinary open access archive for the deposit and dissemination of scientific research documents, whether they are published or not. The documents may come from teaching and research institutions in France or abroad, or from public or private research centers.
L'archive ouverte pluridisciplinaire HAL, est destinée au dépôt et à la diffusion de documents scientifiques de niveau recherche, publiés ou non, émanant des établissements d'enseignement et de recherche français ou étrangers, des laboratoires publics ou privés. 


\title{
A switching technique for output feedback stabilization at an unobservable target
}

\author{
Lucas Brivadis and Ludovic Sacchelli
}

\begin{abstract}
We consider the problem of dynamic output feedback stabilization at an unobservable target point. The challenge lies in according the antagonistic nature of the objective and the properties of the system: the system tends to be less observable as it approaches the target. In the literature, switching techniques rapidly appeared as a suitable approach to deal with this issue. On a case of systems with linear conservative dynamics and nonlinear output, this approach is used in conjunction with an embedding into bilinear systems that admit observers with dissipative error. Combining these two elements, global stabilization by means of a dynamic periodic time-varying output feedback is proved, and numerical simulations are provided.
\end{abstract}

\section{INTRODUCTION}

Stabilizing a control system by using only a measured output is a challenging issue occurring in many practical situations. On nonlinear systems, a general strategy known as observer-based design consists in finding a stabilizing state feedback, designing an observer system that learns the state from the dynamics of its output, and using as an input the state feedback applied to the observer. In [18], [19], Teel and Praly proved under a complete uniform observability assumption that semiglobal smooth stationary dynamic output feedback stabilization can be achieved thanks to this observer-based strategy. Uniform observability refers to the fact that all the inputs render the system observable. However, nonlinear systems are generically non-uniformly observable as soon as the dimension of the output is less or equal to the dimension of the input (see [9], [10]), i.e., there generically exists singular inputs making the system unobservable.

This issue, important from both the theoretical [3], [4], [6] and practical [1], [12], [14], [17] viewpoints, becomes especially salient when the constant input corresponding to the value of the state feedback at the target point renders the system unobservable. In that case, stateestimation and stabilization objectives work against each other: while the system approaches the target, observability of the system worsens, leading to a potential loss

This research was partially funded by the French Grant ANR ODISSE (ANR-19-CE48-0004-01)

L. Brivadis and L. Sacchelli are with Univ. Lyon, Université Claude Bernard Lyon 1, CNRS, LAGEPP UMR 5007, 43 bd du 11 novembre 1918, F-69100 Villeurbanne, France lucas.brivadis@univ-lyon1.fr, ludovic.sacchelli@univ-lyon1.fr in the quality of the state estimation, and in turn, the stabilization.

In [7], Coron introduced a new strategy by allowing the output feedback to be time-varying. Doing so, local output feedback stabilization was achieved up to a Lie nullobservability condition by using a switching strategy: the input is alternatively chosen to estimate or stabilize the state. Later, Shim and Teel in [16] applied a similar principle to obtain practical semi-global stabilization of general systems that can have poor observability guarantees. Nevertheless, a complete method for exact semiglobal (or global) stabilization using these techniques remains to be identified.

In [7], the one-dimensional nonlinear system $\dot{x}=u$, $y=x^{2}$ was investigated: it was proved to be unobservable at the target (i.e. for $u \equiv 0$ ) and not (locally) stabilizable by means of a stationary dynamic output feedback, but locally stabilizable by means of a dynamic periodic timevarying output feedback. In this paper, we investigate an extension of this illustrative example in higher dimension such that its unobservability property at the target is preserved. Interestingly, it has been shown in [4] that the system is stabilizable by means of a stationary dynamic output feedback in some cases. Yet, in others, one is forced to look for time-varying output feedbacks, which is the aim of this paper. In Section II, we recall definitions of output feedback stabilizability. Systems under consideration are discussed in [II], where the result of [4] is recalled. The main result of this paper is stated and proved in Section IV. Numerical simulations are provided in the last section of the paper.

\section{Definitions}

Consider the nonlinear observed control system

$$
\left\{\begin{array}{l}
\dot{x}=f(x, u) \\
y=h(x)
\end{array}\right.
$$

where $x \in \mathbb{R}^{n}$ is the state of the system, $y \in \mathbb{R}^{m}$ is the measured output and $u \in \mathbb{R}^{p}$ is the control, or input. The usual notion of stationary dynamic output feedback stabilization (see, e.g., [2]) is the following.

Definition 1 (Stationary stabilizability): System (II.1) is said to be locally (resp. globally) stabilizable by means of a stationary dynamic output feedback if and only if there exist two continuous maps $\nu: \mathbb{R}^{q} \times \mathbb{R}^{p} \times \mathbb{R}^{m} \rightarrow$ $\mathbb{R}^{q}$ and $\lambda: \mathbb{R}^{q} \times \mathbb{R}^{m} \rightarrow \mathbb{R}^{p}$ for some positive integer $q$ such that $(0,0) \in \mathbb{R}^{n} \times \mathbb{R}^{q}$ is a locally (resp. globally) 
asymptotically stable equilibrium point of the following system:

$$
\left\{\begin{array}{l}
\dot{x}=f(x, u) \\
y=h(x)
\end{array}, \quad\left\{\begin{array}{l}
\dot{\hat{z}}=\nu(\hat{z}, u, y) \\
u=\lambda(\hat{z}, y)
\end{array}\right.\right.
$$

Remark 1: We have assumed with no loss of generality (up to a change of coordinates), that the target point at which we aim to stabilize the state of (II.1) is $x=0$. Moreover, if (II.1) is locally stabilizable by means of a stationary dynamic output feedback, then $f\left(0, u^{\star}\right)=0$, where $u^{\star}=\lambda(0, h(0))$ is the value of the control at the target point. Again, with no loss of generality, we assume that $u^{\star}=0$.

\section{Definition 2 (Observability at the target):}

System (II.1) is said to be observable at the target in some time $T>0$ if and only if, for any pair of trajectories $\left(x_{1}, x_{2}\right)$ of (II.1) with $u \equiv 0$ with corresponding outputs $\left(y_{1}, y_{2}\right)$ defined in $[0, T]$,

$$
y_{1} \equiv y_{2} \Longrightarrow x_{1} \equiv x_{2}
$$

where " $\equiv$ " denotes equality over $[0, T]$.

In this paper, we focus on systems that are unobservable at the target point. The periodic time-varying dynamic output feedbacks considered in [7], [13] or [16] all fit the following extension.

Definition 3 (Periodic time-varying stabilizability): System (II.1) is said to be locally (resp. globally) stabilizable by means of a dynamic periodic timevarying output feedback if and only if there exist $\mu: \mathbb{R}^{q} \times \mathbb{R}^{m} \rightarrow \mathbb{R}^{q}$ and two $T$-periodic maps $\nu: \mathbb{R}^{q} \times \mathbb{R}^{p} \times \mathbb{R}^{m} \times \mathbb{R}_{+} \rightarrow \mathbb{R}^{q}$ and $\lambda: \mathbb{R}^{q} \times \mathbb{R}^{m} \times \mathbb{R}_{+} \rightarrow \mathbb{R}^{p}$, continuous on their spatial domain and piecewise continuous on $\mathbb{R}_{+}$, such that $(0,0) \in \mathbb{R}^{n} \times \mathbb{R}^{q}$ is a locally (resp. globally) asymptotically stable equilibrium point of the following system:

$$
\left\{\begin{array}{l}
\dot{x}=f(x, u) \\
y=h(x)
\end{array}, \quad\left\{\begin{array}{l}
\dot{\hat{z}}=\nu(\hat{z}, u, y, t) \quad t \in\left[t_{k}, t_{k+1}\right) \\
\hat{z}\left(t_{k}\right)=\mu\left(\hat{z}\left(t_{k}^{-}\right), y\left(t_{k}^{-}\right)\right) \\
u=\lambda(\hat{z}, y, t)
\end{array}\right.\right.
$$

where $t_{k}=k T, k \in \mathbb{N}, T>0$, and $\left(\hat{z}\left(t_{k}^{-}\right), y\left(t_{k}^{-}\right)\right)$stands for $\lim _{\substack{t \rightarrow k T \\ t<k T}}(\hat{z}(t), y(t))$ for $k \geqslant 1$.

Remark 2: In Definition 3, $\mu$ may be chosen such that $\mu(\hat{z}, y)=\hat{z}$ for all $\hat{z} \in \mathbb{R}^{q}$ and $y \in \mathbb{R}^{m}$. In that case, (II.4) is simply an ordinary differential equation (ODE). It is the case in [16] for example. However, in this paper, $\hat{z}$ is reinitialized at the beginning of each time period. Yet, the problem under consideration is still the output feedback stabilization since $\hat{z}\left(t_{k}\right)$ depends only on the limits from below of $\hat{z}$ and $y$ on the previous time interval. System (II.4) can also be written as a cascade of ODEs.

\section{A CLASS OF SYSTEMS UNOBSERVABLE AT THE TARGET}

As stated in the introduction, inspired by the onedimensional example $\dot{x}=u, y=x^{2}$ (see [7]), we look for a natural extension of this system in higher dimension. Throughout the paper, we investigate the output feedback stabilizability of the following singleinput single-output (SISO) system:

$$
\left\{\begin{array}{l}
\dot{x}=A x+B u \\
y=\frac{1}{2}|x|^{2}
\end{array}, \quad A^{\prime}=-A\right.
$$

where $A \in \mathbb{R}^{n \times n}$ is a skew-symmetric matrix ( $A^{\prime}$ denotes the transpose of $A), B \in \mathbb{R}^{n \times 1}$ and $|x|$ denotes the Euclidean norm of $x$.

Remark 3: Up to a change of scalar product, one may also consider any system of the form $\dot{x}=A x+B u, y=$ $x^{\prime} P x$ if $A^{\prime} P+P A=0$ for some symmetric positivedefinite matrix $P \in \mathbb{R}^{n \times n}$.

Clearly, (III.1) is unobservable at the target. Indeed, if $x_{a}, x_{b}$ in $\mathbb{R}^{n}$ are such that $\left|x_{a}\right|=\left|x_{b}\right|$, then the solutions of $\dot{x}=A x$ starting from $x_{a}$ and $x_{b}$ are such that their corresponding outputs $y_{a}$ and $y_{b}$ are constantly equal since $A$ is skew-symmetric. In a recent work [4], the cases where (III.1) is stabilizable by means of a stationary dynamic output feedback have been fully characterized.

Theorem 1 (see [4]): Let $A \in \mathbb{R}^{n \times n}$ be a skewsymmetric matrix and $B \in \mathbb{R}^{n \times 1}$.

(i) If $A$ is not invertible, then (III.1) is not locally stabilizable by means of a stationary dynamic output feedback.

(ii) On the other hand, if $A$ is invertible and $(A, B)$ is controllable, then (III.1) is semi-globally stabilizable by means of a stationary dynamic output feedback.

Remark 4: Since $A$ is skew-symmetric, $A$ can be invertible only if $n$ is even. In particular, if $n=1$, (i) states the same result as [7].

We state the proof of (i) to underline how the invertibility of $A$ plays a role in the stabilizability of the system. We also give a step of the proof of (ii) that is used in proof of the main result of the present paper.

Proof of (i): Assume that $(0,0)$ is a locally asymptotically stable equilibrium point of (II.2) with $f(x, u)=$ $A x+B u$ and $h(x)=\frac{1}{2}|x|^{2}$. Let $F$ be the vector field associated to the closed-loop system (II.2), that is, $F(x, \hat{z})=(A x+B \lambda(\hat{z}, h(x)), \nu(\hat{z}, \lambda(\hat{z}, h(x)), h(x)))$. Then, according to Theorem 52.1 in [11] (see [8] when one does not have uniqueness of the solutions to the Cauchy problem), the index of $-F$ at $(0,0)$ is 1 . Assume, for the sake of contradiction, that $A$ is not invertible. Let $\mathcal{N}$ be a one-dimensional subspace of $\operatorname{ker} A$. Denote by $\Sigma$ the reflection through the hyperplane $\mathcal{N}^{\perp}$, that is, $\Sigma=\mathrm{id}_{n}-2 v v^{\prime}$ for some unitary vector $v \in \mathcal{N}$. Then $\operatorname{det} \Sigma=-1, A \Sigma=A$ and $h(\Sigma x)=h(x)$. Hence $(x, \hat{z}) \mapsto$ $-F(\Sigma x, \hat{z})$ has index -1 at $(0,0)$ and $F(\Sigma x, \hat{z})=F(x, \hat{z})$. Thus $1=-1$ which is a contradiction.

Remark 5: This proof emphasizes that the invertibility of $A$ is a topological constraint on the vector field rather than an observability constraint: the unobservability of the system for $u \equiv 0$ does not play any role in the proof. Hence, when $A$ is invertible, stationary stabilizability can be reached, as stated in (ii). Moreover, in all cases, 
considering time-varying systems remove the topological constraint. Hence time-varying output feedback stabilization can be considered on system (III.1.

One step of the proof of (ii): In previous works [5], [15] the role of dissipative systems in the context of output feedback stabilization has been emphasized, which led us to consider on non-dissipative systems embeddings into dissipative ones. A crucial step of the proof of (ii) is to embed the original system (III.1) into a new bilinear system for which an observer with dissipative error can be built. More precisely, let us consider the mapping

$$
\begin{aligned}
\tau: \mathbb{R}^{n} & \longrightarrow \mathbb{R}^{n+1} \\
x & \longmapsto\left(x, \frac{1}{2}|x|^{2}\right)
\end{aligned}
$$

For all $z=\left(z_{1}, \ldots, z_{n+1}\right) \in \mathbb{R}^{n+1}$, define $\bar{z}_{n}=$ $\left(z_{1}, \ldots, z_{n}\right) \in \mathbb{R}^{n}$. If $x$ is a solution of (III.1), then $\frac{1}{2} \frac{\mathrm{d}}{\mathrm{d} t}|x|^{2}=x^{\prime} A x+u B^{\prime} x=u B^{\prime} x$ since $A$ is skewsymmetric. Hence $z=\tau(x)$ defines an embedding of (III.1) into

$$
\left\{\begin{array}{l}
\dot{z}=\mathcal{A}(u) z+\mathcal{B} u \\
y=\mathcal{C} z
\end{array}\right.
$$

where $\mathcal{A}(u)=\left(\begin{array}{cc}A & 0 \\ u B^{\prime} & 0\end{array}\right), \mathcal{B}=\left(\begin{array}{c}B \\ 0\end{array}\right)$ and $\mathcal{C}=$ $\left(\begin{array}{llll}0 & \cdots & 0 & 1\end{array}\right)$ and with initial conditions in $\mathcal{T}=\tau\left(\mathbb{R}^{n}\right)$. Moreover, the semi-trajectory $z$ remains in $\mathcal{T}$.

Let us introduce a Luenberger observer with dynamic gain for (III.3). In order to make the error system dissipative, set $\mathcal{L}_{\alpha}(u)=\left(\begin{array}{c}B u \\ \alpha\end{array}\right) \in \mathbb{R}^{n+1}$ for some positive constant $\alpha$ (degree of freedom). The corresponding observer system is given by

$$
\left\{\begin{array}{l}
\dot{\varepsilon}=\left(\mathcal{A}(u)-\mathcal{L}_{\alpha}(u) \mathcal{C}\right) \varepsilon \\
\dot{\hat{z}}=\mathcal{A}(u) \hat{z}+\mathcal{B} u-\mathcal{L}_{\alpha}(u) \mathcal{C} \varepsilon
\end{array}\right.
$$

where $z=\hat{z}-\varepsilon$ satisfies (III.3), $\hat{z}$ is the estimation of the state made by the observer system and $\varepsilon$ is the error between the estimation of the state and the actual state of the system. The $n$ first coordinates of $\hat{z}$ correspond to an observer of the original system (III.1). Note that for all $u \in \mathbb{R}$,

$$
\mathcal{A}(u)-\mathcal{L}_{\alpha}(u) \mathcal{C}=\left(\begin{array}{cc}
A & -B u \\
u B^{\prime} & 0
\end{array}\right)-\alpha \mathcal{C}^{\prime} \mathcal{C},
$$

that is, the sum of two matrices, one skew-symmetric and one semi-negative definite. It implies that the $\varepsilon$ subsystem of (III.4 is dissipative, that is, for all input $u \in C^{0}\left(\mathbb{R}_{+}, \mathbb{R}\right)$, the solutions of (III.4) satisfy

$$
\frac{\mathrm{d}|\varepsilon|^{2}}{\mathrm{~d} t}=2 \varepsilon^{\prime}\left(\mathcal{A}(u)-\mathcal{L}_{\alpha}(u) \mathcal{C}\right) \varepsilon=-2 \alpha|\mathcal{C} \varepsilon|^{2} \leqslant 0 .
$$

This property allows to mitigate the observability issue of the system. Even if the input approaches a value making the system unobservable, the error between the state estimation and the actual state will not increase. This a key argument in the proof of Theorem 1 (ii), as well as the proof of the main result below.
Remark 6: For any constant $u \in \mathbb{R} \backslash\{0\}$, the pair $(\mathcal{C}, \mathcal{A}(u))$ is observable. Indeed, the Kalman observability matrix $O_{\mathcal{C}, \mathcal{A}(u)}$ of the pair has shape

$$
\left(\begin{array}{cc}
0 & 1 \\
O_{B^{\prime}, A} & 0
\end{array}\right)
$$

where $O_{B^{\prime}, A}$ is the Kalman observability matrix of the pair $\left(B^{\prime}, A\right)$. As such, $O_{B^{\prime}, A}$ is the transposed Kalman controllability matrix of $(-A, B)$, thus invertible. Hence, for any $u \in \mathbb{R} \backslash\{0\}, \mathcal{A}(u)-\mathcal{L}_{\alpha}(u) \mathcal{C}$ is Hurwitz. Indeed, according to (III.6) and LaSalle's invariance principle, the $\omega$-limit set of $\varepsilon$ is a subset of $\operatorname{ker} \mathcal{C}$ invariant under the dynamics $\dot{\varepsilon}=\left(\mathcal{A}(u)-\mathcal{L}_{\alpha}(u) \mathcal{C}\right) \varepsilon$. But since the pair $(\mathcal{C}, \mathcal{A}(u))$ is observable, this set is $\{0\}$, i.e., $\varepsilon \rightarrow 0$, i.e., $\mathcal{A}(u)-\mathcal{L}_{\alpha}(u) \mathcal{C}$ is Hurwitz.

\section{MAin RESUlt}

We have seen in the previous section that semi-global stationary dynamic output feedback stabilization can be achieved as soon as a $A$ is invertible. Combining the embedding-based observer (III.4) with a switching strategy inspired by [7], [16], we show that in all cases, global dynamic time-varying output feedback stabilization can be achieved.

Theorem 2: Let $A \in \mathbb{R}^{n \times n}$ be a skew-symmetric matrix and $B \in \mathbb{R}^{n \times 1}$ such that $(A, B)$ is controllable. Then (III.1) is globally stabilizable by means of a dynamic periodic time-varying output feedback.

Remark 7: The results of [7] imply the local stabilizability of (III.1) (in finite-time), while [16] implies the semi-global practical stabilizability, that is, stabilizability in an arbitrarily small neighborhood of the target for initial conditions in an arbitrarily large compact set of initial conditions. Theorem 2 bridges the gap between these two results on the specific class of examples (III.1), since global exact stabilization is obtained.

Remark 8: Since $A$ is skew-symmetric, $(A, B)$ is controllable if and only if it is stabilizable due to the Hautus tests. Indeed, all the eigenvalues of $A$ have null real part, hence the Hautus tests for controllability and stabilizability are equivalent. Moreover, such pairs are easily characterizable using this test and the skew-symmetry of $A$.

In this section, we prove the main Theorem 2 by defining a dynamic periodic time-varying output feedback that globally stabilizes system (III.1). Let $T=$ $T_{\text {obs }}+T_{\text {stab }}>0$ be the period of the feedback to be fixed later. Let $t_{k}=k T$ for all $k \in \mathbb{N}$. The closed-loop system can be written as (II.4), with $f(x, u)=A x+B u$ and $h(x)=\frac{1}{2}|x|^{2}$. The strategy is the following one:

$A$. During time intervals $\left[t_{k}, t_{k}+T_{\text {obs }}\right], k \in \mathbb{N}$, called observation phases, one excites system (III.1) with a constant control that is null at the equilibrium point. This excitation will guarantee that the observer error tends towards 0 , since all non-zero constant inputs make the system observable. The $\hat{z}$-part of 
system (II.4 is chosen to be a Luenberger-type observer of the embedded system defined in (III.4).

$B$. During time intervals $\left[t_{k}+T_{\text {obs }}, t_{k+1}\right], k \in \mathbb{N}$, called stabilization phases, one stabilizes system (III.1) by applying a stabilizing state feedback to the estimation of the state obtained by the observer. The $\hat{z}$ part of system (II.4) is chosen to be a copy of the $z$-dynamics.

In the two following sections, we define more precisely the feedback law $\lambda(\hat{z}, y, t)$ on the different phases.

\section{A. Observation phase}

Let $k \in \mathbb{N}$. For all $t \in\left[t_{k}, t_{k}+T_{\text {obs }}\right]$, define

$$
u=\lambda(\hat{z}, y, t):=\sqrt{y\left(t_{k}\right)}+\frac{1}{\sqrt{2}}\left|\bar{z}_{n}\right|\left(t_{k}\right)
$$

Remark 9: The key argument in the proof of asymptotic stability will be that $u$ vanishes if and only if the closed-loop system is at the target point, i.e., $\left(x\left(t_{k}\right), \hat{z}\left(t_{k}\right)\right)=(0,0)$. There lies the gap between $[7]$ and [16]: only practical stabilization was obtained in [16] because the input during observation phases does not vanish at the target. On the contrary, the observable input chosen in [7] vanishes at the target, but only local stabilization is obtained due to the lack of Lyapunov analysis (as in [16]) or dissipativity properties (as in the present paper).

The closed-loop on $\left[t_{k}, t_{k}+T_{\text {obs }}\right]$ can be written as

$$
\left\{\begin{array}{l}
\dot{\varepsilon}=\left(\mathcal{A}(u)-\mathcal{L}_{\alpha}(u) \mathcal{C}\right) \varepsilon \\
\dot{\hat{z}}=\mathcal{A}(u) \hat{z}+\mathcal{B} u-\mathcal{L}_{\alpha}(u) \mathcal{C} \varepsilon
\end{array}\right.
$$

The observer is initialized in the following manner:

- If $k=0$, then $\hat{z}(0), \varepsilon(0) \in \mathbb{R}^{n+1}, \hat{z}_{n+1}(0)=y(0)$ and $\varepsilon_{n+1}(0)=0$.

- Otherwise, $\hat{z}_{n+1}\left(t_{k}\right)=y\left(t_{k}\right), \varepsilon_{n+1}\left(t_{k}\right)=0, \overline{\hat{z}}_{n}\left(t_{k}\right)=$ $\overline{\hat{z}}_{n}\left(t_{k}^{-}\right)$and $\bar{\varepsilon}_{n}\left(t_{k}\right)=\bar{\varepsilon}_{n}\left(t_{k}^{-}\right)$.

The trajectory $x$ over $\left[t_{k}, t_{k}+T_{\text {obs }}\right]$ starting from $x_{0}$ if $k=0$, and $\overline{\hat{z}}_{n}\left(t_{k}\right)-\bar{\varepsilon}_{n}\left(t_{k}\right)$ otherwise, satisfying

$$
\dot{x}=A x+B u,
$$

is such that $x=\overline{\hat{z}}_{n}-\bar{\varepsilon}_{n}$. According to the variation of constant formula,

$$
x\left(t_{k}+T_{\mathrm{obs}}\right)=e^{T_{\mathrm{obs}} A} x\left(t_{k}\right)+\int_{0}^{T_{\mathrm{obs}}} e^{\left(T_{\mathrm{obs}}-s\right) A} B u \mathrm{~d} s .
$$

Hence, since $A$ is skew-symmetric,

$$
\begin{aligned}
\left|x\left(t_{k}+T_{\text {obs }}\right)\right| \leqslant & \left|x\left(t_{k}\right)\right|+T_{\text {obs }} \frac{|B|}{\sqrt{2}}\left(\left|x\left(t_{k}\right)\right|+\left|\bar{z}_{n}\left(t_{k}\right)\right|\right) \\
\leqslant( & \left.+T_{\text {obs }} \sqrt{2}|B|\right)\left|x\left(t_{k}\right)\right| \\
& +T_{\text {obs }} \frac{|B|}{\sqrt{2}}\left|\bar{\varepsilon}_{n}\left(t_{k}\right)\right|
\end{aligned}
$$

since $\overline{\hat{z}}_{n}=\bar{\varepsilon}_{n}+x$. Thus, with $T_{\mathrm{obs}}=\frac{\sqrt{2}}{|B|}$,

$$
\left|x\left(t_{k}+T_{\text {obs }}\right)\right| \leqslant 3\left|x\left(t_{k}\right)\right|+\left|\bar{\varepsilon}_{n}\left(t_{k}\right)\right| .
$$

Moreover, according to (III.6), for all $t \in\left[t_{k}, t_{k}+T_{\mathrm{obs}}\right]$,

$$
\left|\bar{\varepsilon}_{n}(t)\right| \leqslant|\varepsilon(t)| \leqslant\left|\varepsilon\left(t_{k}\right)\right|=\left|\bar{\varepsilon}_{n}\left(t_{k}\right)\right| .
$$

\section{B. Stabilization phase}

Since $(A, B)$ is controllable, there exists $K \in \mathbb{R}^{1 \times n}$ be such that $A-B K$ is Hurwitz. Let $k \in \mathbb{N}$. For all $t \in\left[t_{k}+T_{\text {obs }}, t_{k+1}\right]$, define

$$
u=\lambda(\hat{z}, y, t):=-K \overline{\hat{z}}_{n}
$$

The closed-loop on $\left[t_{k}+T_{\text {obs }}, t_{k+1}\right]$ can be written as

$$
\left\{\begin{array}{l}
\dot{\varepsilon}=\mathcal{A}(u) \varepsilon \\
\dot{\hat{z}}=\mathcal{A}(u) \hat{z}-\mathcal{B} K \overline{\hat{z}}_{n}
\end{array}\right.
$$

initialized at $\hat{z}\left(t_{k}+T_{\text {obs }}\right)=\hat{z}\left(\left(t_{k}+T_{\text {obs }}\right)^{-}\right)$and $\varepsilon\left(t_{k}+\right.$ $\left.T_{\text {obs }}\right)=\varepsilon\left(\left(t_{k}+T_{\text {obs }}\right)^{-}\right)$. Using the definition of the embedded system (III.3), we find

$$
\dot{\bar{\varepsilon}}_{n}=A \bar{\varepsilon}_{n}, \quad \dot{\overline{\hat{z}}}_{n}=A \overline{\hat{z}}_{n}+B u
$$

Note that $\dot{\bar{\varepsilon}}_{n}$ and $\dot{\overline{\hat{z}}}_{n}$ have a stationary dynamics, and $\lambda(\hat{z}, y, t)$ depends only on $\overline{\hat{z}}_{n}$. Moreover, since $A$ is skewsymmetric, we have for all $t \in\left[t_{k}+T_{\mathrm{obs}}, t_{k+1}\right]$,

$$
\left|\bar{\varepsilon}_{n}(t)\right|=\left|\bar{\varepsilon}_{n}\left(t_{k}+T_{\text {obs }}\right)\right| .
$$

The trajectory $x$ over $\left[t_{k}+T_{\mathrm{obs}}, t_{k+1}\right]$ starting from $\overline{\hat{z}}_{n}\left(t_{k}+T_{\text {obs }}\right)-\bar{\varepsilon}_{n}\left(t_{k}+T_{\text {obs }}\right)$, satisfying

$$
\begin{aligned}
\dot{x} & =A x+B u \\
& =(A-B K) x-B K\left(\overline{\hat{z}}_{n}-x\right)
\end{aligned}
$$

is such that $x=\overline{\hat{z}}_{n}-\bar{\varepsilon}_{n}$. Then

$$
\begin{aligned}
& x\left(t_{k+1}\right)=e^{T_{\text {stab }}(A-B K)} x\left(t_{k}+T_{\text {obs }}\right) \\
& \quad-\int_{0}^{T_{\text {stab }}} e^{\left(T_{\text {stab }}-t\right)(A-B K)} B K \bar{\varepsilon}_{n}\left(t_{k}+T_{\text {obs }}+t\right) \mathrm{d} t .
\end{aligned}
$$

Since $A-B K$ is Hurwitz, there exists $T_{\text {stab }}>0$ large enough such that $\left\|e^{(A-B K) T_{\text {stab }}}\right\| \leqslant \frac{1}{4}$. Then, according to (IV.9),

$$
\left|x\left(t_{k+1}\right)\right| \leqslant \frac{1}{4}\left|x\left(t_{k}+T_{\text {obs }}\right)\right|+\gamma\left|\bar{\varepsilon}_{n}\left(t_{k}+T_{\text {obs }}\right)\right|
$$

with $\gamma=\left\|\int_{0}^{T_{\text {stab }}} e^{\left(T_{\text {stab }}-t\right)(A-B K)} B K \mathrm{~d} t\right\|$.

\section{Attractivity}

With the definition of the dynamic periodic timevarying output feedback given in the observation and stabilization phases, we now prove that for any initial conditions $x_{0} \in \mathbb{R}^{n}$ and $\hat{z}_{0} \in \mathbb{R}^{n+1}$ such that $\hat{z}_{n+1}(0)=$ $\frac{1}{2}\left|x_{0}\right|^{2}$, the corresponding solution $(x, \hat{z})$ tends towards zero. Combining (IV.5) and (IV.9),

$$
\left|\bar{\varepsilon}_{n}(t)\right| \leqslant\left|\bar{\varepsilon}_{n}\left(t_{k}\right)\right|
$$

for all $t \in\left[t_{k}, t_{k+1}\right]$. Hence (IV.4) and (IV.11) yields

$$
\left|x\left(t_{k+1}\right)\right| \leqslant \frac{3}{4}\left|x\left(t_{k}\right)\right|+\left(\gamma+\frac{1}{4}\right)\left|\bar{\varepsilon}_{n}\left(t_{k}\right)\right|
$$

We recognize an Input-to-State Stability (ISS) property. By (IV.12), $\left(\left|\bar{\varepsilon}_{n}\left(t_{k}\right)\right|\right)_{k \in \mathbb{N}}$ is a bounded sequence. Hence $\left(\left|x\left(t_{k}\right)\right|\right)_{k \in \mathbb{N}}$ is also bounded. Over the observation and 
stabilization phases, $\dot{x}=A x+B u$ with $u$ a continuous function of time. Hence, the corresponding flows are continuous with respect to the initial conditions of the system. Thus $x, \overline{\hat{z}}_{n}$ and $u$ are bounded functions of time.

Let us prove by contradiction that $\bar{\varepsilon}_{n}\left(t_{k}\right) \rightarrow 0$. Assume the contrary. Then $\left|x\left(t_{k}\right)\right|$ or $\left|\overline{\hat{z}}_{n}\right|$ does not converge to 0 . In both cases, $u\left(t_{k}\right)$ does not converge to 0 (by (IV.1)). Since $u$ is bounded, up to an extraction, there exists $u^{\star} \neq$ 0 such that $u\left(t_{k}\right) \rightarrow u^{\star}$ as $k \rightarrow+\infty$. We have

$$
\begin{aligned}
\left|\bar{\varepsilon}_{n}\left(t_{k+1}\right)\right| & =\left|\bar{\varepsilon}_{n}\left(t_{k}+T_{\text {obs }}\right)\right| \\
& \leqslant\left\|e^{T_{\text {obs }}\left(\mathcal{A}\left(u\left(t_{k}\right)\right)-\mathcal{L}_{\alpha}\left(u\left(t_{k}\right)\right) \mathcal{C}\right)}\right\|\left|\bar{\varepsilon}_{n}\left(t_{k}\right)\right| .
\end{aligned}
$$

Since $\mathcal{A}\left(u^{\star}\right)-\mathcal{L}_{\alpha}\left(u^{\star}\right) \mathcal{C}$ is Hurwitz (see Remark 6), there exists $k_{0} \in \mathbb{N}$ such that for all $k \geqslant k_{0}$, $\left\|e^{T_{\mathrm{obs}}\left(\mathcal{A}\left(u\left(t_{k}\right)\right)-\mathcal{L}_{\alpha}\left(u\left(t_{k}\right)\right) \mathcal{C}\right)}\right\| \leqslant \mu$ for some $\mu \in(0,1)$. Hence $\bar{\varepsilon}_{n}\left(t_{k}\right) \rightarrow 0$, which is a contradiction.

Thus, $\bar{\varepsilon}_{n}\left(t_{k}\right) \rightarrow 0$ as $k \rightarrow+\infty$. According to the ISS property (IV.13), $\left|x\left(t_{k}\right)\right| \rightarrow 0$ as $k$ goes to infinity. Since the flows are continuous over the observation and stabilization phases, $x(t) \rightarrow 0$ and $\bar{\varepsilon}_{n}(t) \rightarrow 0$ as $t \rightarrow \infty$. Moreover, $\varepsilon_{n+1}\left(t_{k}\right)=0$ for all $k \in \mathbb{N}$. Hence $\varepsilon(t) \rightarrow 0$. Finally, $x(t) \rightarrow 0$ and $\hat{z}(t) \rightarrow 0$ as $t \rightarrow \infty$.

\section{Stability}

The attractivity being proved in the previous section, it remains to show the Lyapunov stability of the closedloop system. Let $R>0$. Set $r=\min \left(\frac{R}{4(4 \gamma+1)}, \frac{R}{16}\right)$. Assume that $\left|x_{0}\right| \leqslant r$ and $\left|\hat{z}_{0}\right| \leqslant r$. Then $\left|\varepsilon_{0}\right| \leqslant 2 r$ and, by (IV.12), $\left|\varepsilon\left(t_{k}\right)\right| \leqslant 2 r$ for all $k \in \mathbb{N}$. Moreover, $\frac{3}{4} R+2 r\left(\gamma+\frac{1}{4}\right) \leqslant \frac{7}{8} R$. Hence, using (IV.13) by induction for $k \in \mathbb{N},\left|x\left(t_{k}\right)\right| \leqslant \frac{7}{8} R \leqslant R$ for all $k \in \mathbb{N}$. Moreover, $\left|\hat{z}\left(t_{k}\right)\right| \leqslant\left|x\left(t_{k}\right)\right|+\left|\varepsilon\left(t_{k}\right)\right| \leqslant \frac{7}{8} R+2 \frac{R}{16}=R$. Thus $\left|x\left(t_{k}\right)\right| \leqslant$ $R$ and $\left|\hat{z}\left(t_{k}\right)\right| \leqslant R$ for all $k \in \mathbb{N}$. Since the flows are continuous over the observation and stabilization phases, the system is Lyapunov stable at $(x, \hat{z})=(0,0)$.

\section{Numerical Simulation}

We propose a numerical simulation of the stabilization strategy of Theorem 2. In dimension $n=2$, we choose $A=\left(\begin{array}{cc}0 & -1 \\ 1 & 0\end{array}\right)$ and $b=\left(\begin{array}{l}0 \\ 1\end{array}\right)$, which is a controllable pair. The parameters of the dynamic periodic time-varying output feedback are chosen according to Table @. The initial conditions are $x_{0}=(1,0)$ and $\hat{z}_{0}=(\sqrt{2}, \sqrt{2}, 1 / 2)$, that fit the conditions $T_{\text {obs }}=\frac{\sqrt{2}}{|B|}$ and $\left\|e^{(A-B K) T_{\text {stab }}}\right\| \leqslant$ $\frac{1}{4}$. The resulting trajectory $x$ as well as the first two coordinates of $\hat{z}$ (which are an observer of $x$ ) are plotted in Figure 1 for $t \in[0,5 T]$. The switching times are emphasized, as in Figure 2. In accordance with the Luenberger observer design and equation (III.4), the error between the state and the observer of the embedded system is non-increasing, as it can be checked in Figure 2 . Over the time interval $[0,1000 T]$, we plot in Figure 3 the evolution of the square of the state's norm of the closed-loop system, namely, $|x|^{2}+|\hat{z}|^{2}$. According to Theorem [2] and as it can be seen in Figure 3] $|x|^{2}+|\hat{z}|^{2}$ tends towards 0 as time goes to infinity, but no decay rate is guaranteed. This seems directly correlated to the absence of any guarantees on the speed of convergence of the observer (because of the unobservable target). The convergence is only guaranteed thanks to $\omega$-limit sets arguments. This difficulty is apparent in other such works with similar techniques (see, e.g., [12]) and merits further investigation.

\begin{tabular}{|c|c|}
\hline$T_{\mathrm{obs}}=\sqrt{2}$ & $T_{\mathrm{stab}}=5$ \\
\hline$\alpha=1$ & $K=(0,2)$ \\
\hline
\end{tabular}

TABLE I

Parameters of the Numerical Simulation

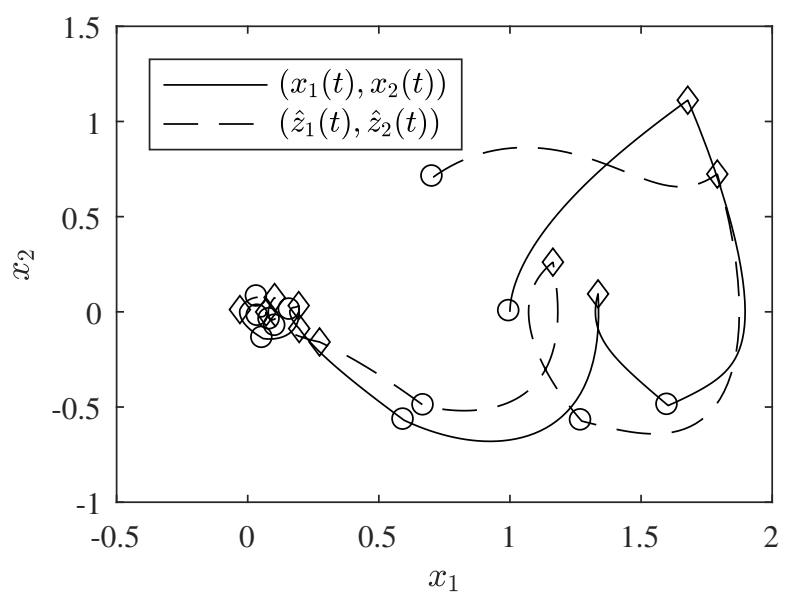

Fig. 1. Trajectory of the closed-loop system starting from $x_{0}=$ $(1,0)$ and $\hat{z}_{0}=(\sqrt{2}, \sqrt{2}, 1 / 2)$ over $[0,5 T]$, i.e., after 5 switches from stabilization phase to observation phase (o) and 5 switches from observation phase to stabilization phase $(\diamond)$.

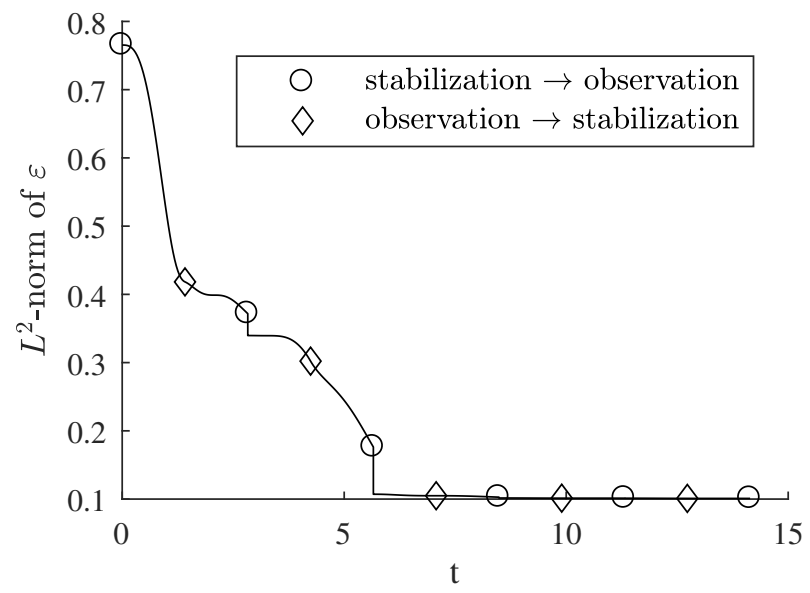

Fig. 2. Evolution of the error norm $|\varepsilon|$ over $[0,5 T]$ where $\varepsilon=$ $\hat{z}-\tau(x)$ and $\tau$ is the embedding defined by (III.2). As stated by (III.4), $|\varepsilon|$ is non-increasing.

\section{CONCLUSION}

We have shown on a class of systems with linear conservative dynamics and nonlinear output that global 


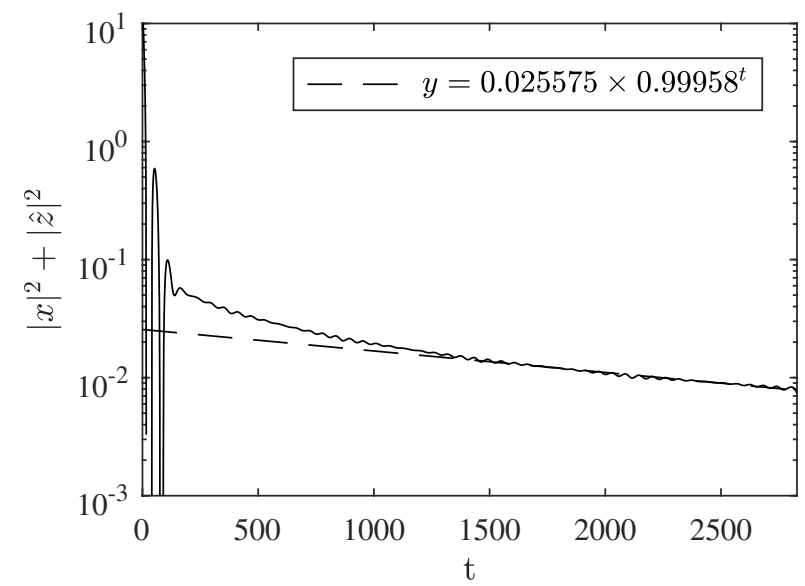

Fig. 3. Evolution of the norm of the state of the closed-loop system. For readability, we only show the upper peak envelope of the signal. The linear regression is obtained on $[1500,3000]$.

dynamic periodic time-varying output feedback stabilization can be achieved. The feedback is obtained as a combination of (i) a Luenberger observer with dissipative error on an embedding of the system; (ii) a switched feedback law alternating between observation phases (during which the observer learns the state) and stabilization phases (during which the state is stabilized). The aim of this paper was to explore the gap between [7] and [16], at least on a specific class of systems. More general switching techniques for semi-global or global exact output feedback stabilization will be investigated in future works.

\section{REFERENCES}

[1] Alain Ajami, Jean-Paul Gauthier, and Ludovic Sacchelli. Dynamic output stabilization of control systems: An unobservable kinematic drone model. Automatica, 125:109383, 2021.

[2] V. Andrieu and L. Praly. A unifying point of view on output feedback designs for global asymptotic stabilization. Automatica. A Journal of IFAC, the International Federation of Automatic Control, 45(8):1789-1798, 2009.

[3] Lucas Brivadis, Jean-Paul Gauthier, Ludovic Sacchelli, and Ulysse Serres. Avoiding observability singularities in output feedback bilinear systems, 2019. Working paper or preprint.

[4] Lucas Brivadis, Jean-Paul Gauthier, Ludovic Sacchelli, and Ulysse Serres. New perspectives on output feedback stabilization at an unobservable target. working paper or preprint, November 2020.

[5] Lucas Brivadis, Ludovic Sacchelli, Vincent Andrieu, Jean-Paul Gauthier, and Ulysse Serres. From local to global asymptotic stabilizability for weakly contractive control systems. Automatica, page 109308, 2020.

[6] P. Combes, A. K. Jebai, F. Malrait, P. Martin, and P. Rouchon. Adding virtual measurements by signal injection. In 2016 American Control Conference (ACC), pages 999-1005, 2016.

[7] Jean-Michel Coron. On the stabilization of controllable and observable systems by an output feedback law. Mathematics of Control, Signals, and Systems, 7(3):187-216, 1994.

[8] Jean-Michel Coron. Relations entre commandabilité et stabilisations non linéaires. In Nonlinear partial differential equations and their applications. Collège de France Seminar, Vol. XI (Paris, 1989-1991), volume 299 of Pitman Research Notes In Mathematics Series, pages 68-86. Longman Scientific \& Technical, Harlow, 1994.
[9] J.-P. Gauthier and I. Kupka. A separation principle for bilinear systems with dissipative drift. Institute of Electrical and Electronics Engineers. Transactions on Automatic Control, 37(12):1970-1974, 1992.

[10] Jean-Paul Gauthier and Ivan Kupka. Deterministic observation theory and applications. Cambridge University Press, Cambridge, 2001.

[11] M. A. Krasnosel'skiı̌ and P. P. Zabreı̌ko. Geometrical methods of nonlinear analysis, volume 263 of Grundlehren der Mathematischen Wissenschaften [Fundamental Principles of Mathematical Sciences]. Springer-Verlag, Berlin, 1984. Translated from the Russian by Christian C. Fenske.

[12] M. Lagache, U. Serres, and J. Gauthier. Exact output stabilization at unobservable points: Analysis via an example. In 2017 IEEE 56th Annual Conference on Decision and Control $(C D C)$, pages 6744-6749, Dec 2017.

[13] D. Nešić and E.D. Sontag. Input-to-state stabilization of linear systems with positive outputs. Systems 83 Control Letters, 35(4):245 - 255, 1998.

[14] A. Rapaport and D. Dochain. A robust asymptotic observer for systems that converge to unobservable states - a batch reactor case study. IEEE Transactions on Automatic Control, 65(6):2693-2699, 2020.

[15] Ludovic Sacchelli, Lucas Brivadis, Vincent Andrieu, Ulysse Serres, and Jean-Paul Gauthier. Dynamic output feedback stabilization of non-uniformly observable dissipative systems, 2020. To appear in IFAC WC 2020.

[16] H. Shim and A.R. Teel. Asymptotic controllability and observability imply semiglobal practical asymptotic stabilizability by sampled-data output feedback. Automatica, 39(3):441 - 454, 2003.

[17] D. Surroop, P. Combes, P. Martin, and P. Rouchon. Adding virtual measurements by pwm-induced signal injection. In 2020 American Control Conference (ACC), pages 2692-2698, 2020.

[18] Andrew Teel and Laurent Praly. Global stabilizability and observability imply semi-global stabilizability by output feedback. Systems \& Control Letters, 22(5):313-325, 1994.

[19] Andrew Teel and Laurent Praly. Tools for semiglobal stabilization by partial state and output feedback. SIAM Journal on Control and Optimization, 33(5):1443-1488, 1995. 